\title{
Impacto de intervenção fisioterapêutica na prevenção do pé diabético
}

\author{
Impact of physical therapy intervention on \\ the prevention of diabetic foot
}

\section{Maria de Fátima Alcântara Barros ${ }^{[a]}$, Jéssyca Carneiro Mendes ${ }^{[b]}$, João Agnaldo do Nascimento ${ }^{[c]}$, Antonio Geraldo Cidrão de Carvalho ${ }^{[\mathrm{d}]}$}

[a] Ph.D. em Santé Publique, Université Montpellier 2, Sciences et Techniques, UM2, França, professora associada do Departamento de Fisioterapia, Laboratório de Fisioterapia em Saúde Coletiva (LabFISC) do Centro de Ciências da Saúde da Universidade Federal da Paraíba (UFPB), João Pessoa, PB - Brasil, e-mail: fatimalcan@yahoo.com

[b] Pesquisadora do Laboratório de Fisioterapia em Saúde Coletiva (LabFISC) da Universidade Federal da Paraíba (UFPB), João Pessoa, PB - Brasil, e-mail: jeh_carneiro@hotmail.com

[c] Professor adjunto do Departamento de Ciências Exatas, Centro de Ciências Aplicadas e Educação - Câmpus IV da Universidade Federal da Paraíba (UFPB), Rio Tinto, PB - Brasil, e-mail: jadnasci@hotmail.com

[d] Ph.D. em Santé Publiqueet Aterogenèse, Université Montpellier 2, Sciences et Techniques, França, professor associado do Departamento de Fisioterapia, Laboratório de Fisioterapia em Saúde Coletiva (LabFISC) do Centro de Ciências da Saúde da Universidade Federal da Paraíba (UFPB), João Pessoa, PB - Brasil, e-mail: gecidrao@yahoo.com.br

\section{Resumo}

Introdução: 0 pé diabético é a complicação mais frequente do diabetes mellitus, caracterizando-se como a principal causa de amputação não traumática dos membros inferiores. Objetivo: Avaliar o impacto de uma intervenção fisioterapêutica na prevenção do pé diabético. Materiais e métodos: Realizou-se um estudo de intervenção com 24 usuários, escolhidos aleatoriamente, no cadastro do Programa de Diabetes do CAIS - Jaguaribe, localizado em João Pessoa (PB). Os dados foram coletados por meio de uma ficha de avaliação fisioterapêutica e de questionários estruturados, aplicados antes e após a intervenção. As análises estatísticas foram realizadas utilizando-se o programa SPSS. Para as variáveis dicotômicas, adotou-se o teste de McNemar, considerando-se estatisticamente significativos os valores de $\mathrm{p}<0,05$. Resultados: Após a intervenção, ocorreram alterações em relação ao hábito de andar sem calçado ( $\mathrm{p}=0,003)$, a examinar os pés com frequência $(p=0,006)$, a não utilizar a prática do escalda-pé $(p=0,013)$, a secar corretamente os 
pés $(\mathrm{p}=0,016)$. Com relação ao uso do calçado apropriado e do emprego da massagem terapêutica nos pés, da hidratação adequada e da detecção e solução de eventuais alterações nos pés, a intervenção teve um impacto relevante $(\mathrm{p}<0,001)$. Todos os usuários $(100 \%)$ consideraram benéficos os exercícios para os pés $(\mathrm{p}<0,001)$, tornando-se um hábito frequente. Conclusão: Os resultados mostraram que a intervenção fisioterapêutica, com ênfase na educação em saúde, foi um instrumento fundamental para conscientizar e modificar hábitos e atitudes que colocavam em risco o pé dos usuários.

Palavras-chave: Diabetes mellitus. Pé diabético. Modalidades de fisioterapia.

\section{Abstract}

Introduction: Diabetic foot is the most common complication of diabetes mellitus, which is characterized as the leading cause of non-traumatic amputation of lower limbs. Objective: To evaluate the impact of a physiotherapy intervention on the prevention of diabetic foot. Materials and methods: An intervention study was carried out with 24 patients, randomly selected, registered in the CAIS Diabetes Program - Jaguaribe, João Pessoa (PB). Data were collected through a physiotherapy assessment form and structured questionnaires, applied before and after intervention. Statistical analyses were performed using SPSS. For dichotomous variables, the McNemar test was adopted, considering $p<0.05$ as statistically significant. Results: After intervention, changes in relation to the habit of walking barefoot ( $p=0.003)$, to examine the feet frequently $(p=0.006)$, not using the practice of hot footbath ( $p=0.013)$ and drying feet properly $(p=0.016)$ were observed. Regarding the use of appropriate footwear and therapeutic massage on feet, proper hydration and the detection and resolution of any changes in the feet, intervention had a significant impact ( $p<0.001)$. All users $(100 \%)$ considered feet exercises beneficial ( $p$ <.001), making it a frequent habit. Conclusion: The results showed that physiotherapy interventions with emphasis on health education was an essential tool to raise awareness and change habits and attitudes that endangered the patients' feet.

Keywords: Diabetes mellitus. Diabetic foot. Physical therapy modalities.

\section{Introdução}

0 diabetes mellitus (DM) configura-se como um problema de saúde pública em ascensão, apresentando elevada morbimortalidade e alto índice de complicações que geram consequências de cunho econômico, social e psicológico, além da diminuição da qualidade de vida dos doentes e seus familiares (1).

0 diabetes é um problema em crescimento na América Latina e no Caribe, esperando-se para os próximos 20 anos a existência de 30 milhões de pessoas vivendo com o diabetes (2). No Brasil, estima-se que o número de diabéticos chegue a 11 milhões até 2025. Segundo publicação da Organização Mundial da Saúde de 2009 (3), as doenças crônicas causam 35 milhões de óbitos a cada ano - ou $60 \%$ de todos os falecimentos em nível mundial, dos quais $80 \%$ ocorrem em países de baixa ou média renda.

Os gastos com o diabetes e suas complicações chegam a US\$ 8,1 bilhões na América Latina e, no Brasil, os custos totais (tanto indiretos como diretos) atingem uma estimativa anual de US\$ 23 milhões. Isso corresponde a um gasto em saúde de aproximadamente US\$ 870 por pessoa (4).

Em longo prazo, o DM acarreta complicações microvasculares, neuropáticas e macrovasculares, em que se destacam as doenças coronarianas, os acidentes vasculares cerebrais e as doenças vasculares periféricas. Além disso, o diabetes mal controlado favorece o desenvolvimento de complicações altamente incapacitantes, principalmente o pé diabético, a cegueira e a insuficiência renal crônica, impedindo as pessoas de continuarem realizando suas atividades diárias e laborais, acarretando alta ocupação de leitos e absenteísmo ao trabalho, pelas internações prolongadas e recorrentes (5).

A neuropatia ocasiona a perda da sensibilidade protetora e, subsequentemente, a deformidade do pé, com possibilidade de uma marcha anormal, tornando o paciente vulnerável a pequenos traumas e lesões de pele causados por calçados impróprios ou por andar descalço (5). 
0 pé diabético representa um estado fisiopatológico multifacetado, caracterizado por úlceras que surgem nos pés, como consequência da neuropatia, em $90 \%$ dos casos (5), representando uma das mais mutilantes complicações crônicas do diabetes mellitus (6), caracterizando-se como a principal causa de amputações não traumáticas de membros inferiores $(7,8)$.

Esse panorama pode ser modificado por meio da implementação de estratégias de educação em saúde que levem conhecimento e informações a respeito dos fatores de risco, das complicações e do autocuidado aos portadores de DM. Tais medidas, se estimadas para os Estados Unidos, levariam à diminuição considerável de $50 \%$ das amputações. Caso fossem estimadas para o Brasil, esse impacto poderia ser ainda maior (9).

Tendo em vista a importância de ações educacionais em saúde, para a prevenção de complicações do diabetes mellitus, foi desenvolvido um estudo de intervenção, com ênfase na educação em diabetes, visando à troca de saberes e vivências, à construção do saber no cuidado com o controle glicêmico, ao aprendizado do autocuidado, principalmente, voltado ao pé diabético. Este trabalho foi desenvolvido com usuários do Sistema Único de Saúde (SUS), em uma unidade de referência em diabetes, no município de João Pessoa (PB), com o intuito de avaliar o impacto de uma intervenção fisioterapêutica na prevenção do pé diabético.

\section{Materiais e métodos}

Realizou-se um estudo de intervenção com usuários do Sistema Único de Saúde, assistidos pelo Programa de Diabetes do Centro de Assistência Integrada à Saúde - CAIS Jaguaribe, localizado em João Pessoa (PB), no período de junho a outubro de 2010. De um total de 362 diabéticos avaliados e que não apresentavam alterações tróficas nos pés, 24 usuários foram selecionados aleatoriamente, por meio de sorteio do prontuário, para a composição da amostra do estudo, tendo a faixa etária variado de 41 a 68 anos.

A coleta de dados foi iniciada por meio de uma avaliação fisioterapêutica, pela qual foram levantadas informações relacionadas ao diabetes, como: o tipo, o tempo de diagnóstico, o tipo de tratamento realizado, a presença de hipertensão arterial associada, calos, queratoses, verrugas, rachaduras, micoses e flictenas, além do aspecto da pele, pilificação, umidade dos pés, tipos de dedos e unhas. A sensibilidade dolorosa, tátil e térmica dos pés foi avaliada utilizando-se instrumentos apropriados. A sensibilidade protetora plantar foi pesquisada por meio da aplicação do monofilamento de Semmes-Weinstein, conforme as recomendações do consenso internacional sobre o pé diabético (10). Por fim, foi analisada a presença dos pulsos tibial posterior e pedioso.

Após a avaliação, os usuários foram submetidos a uma intervenção fisioterapêutica, tendo como objetivo principal a troca de informações e vivências, de modo a construir um saber voltado para o autocuidado do portador de diabetes, buscando, deste modo, a promoção da saúde, a prevenção das complicações inerentes à patologia e a melhoria da qualidade de vida destes indivíduos.

A intervenção fisioterapêutica foi desenvolvida no Serviço de Fisioterapia do CAIS com 24 usuários ( $\mathrm{n}=24)$, divididos em três grupos de oito participantes. Cada grupo foi submetido a oito sessões de tratamento, desenvolvidas por dois fisioterapeutas, uma vez por semana, perfazendo dois meses de intervenção, tendo a sessão 1 hora e 30 minutos.

0 tratamento fisioterapêutico teve a duração de 60 minutos constando de exercícios gerais de alongamento (dez minutos), caminhadas (dez minutos), exercícios do tipo ativo livre e ativo resistido para a flexão plantar, dorsiflexão, inversão e eversão do tornozelo (dez minutos), de flexão, extensão, abdução e adução dos dedos dos pés (dez minutos), utilizando-se bolas, faixas elásticas e bastões para os exercícios ativos livres e pesos de $1 / 2 \mathrm{~kg}$ para os exercícios resistidos. Foram desenvolvidos exercícios para a propriocepção dos pés (dez minutos), empregando-se materiais de diferentes texturas (areia e grãos) e massoterapia superficial e profunda para os pés (dez minutos), além de orientações sobre a marcha.

Em cada encontro, foram destinados 30 minutos para atividade de educação em saúde (orientações gerais: o controle adequado do diabetes, a importância da monitorização da glicemia, complicações agudas e crônicas, estilo de vida saudável, importância da nutrição balanceada, principais cuidados com os pés, utilização de calçados adequados, postura saudável, importância de alongamentos gerais e específicos e de atividades físicas aeróbicas para melhoria da saúde).

Utilizou-se como instrumento para a coleta dos dados uma ficha de avaliação fisioterapêutica e três 
questionários estruturados, abordando aspectos sociodemográficos, da enfermidade, do autocuidado e dos hábitos e atitudes dos usuários, aplicados antes e após a intervenção.

Os dados foram codificados, digitados em dupla entrada e validados utilizando-se o programa EpiInfo, versão 6.04. A análise estatística foi realizada, inicialmente, por meio de descrições tabulares do perfil da amostra e da frequência percentual obtida para cada uma das variáveis de estudo. Para as variáveis dicotômicas, foi adotado o teste de McNemar. Foram considerados estatisticamente significantes os valores de $\mathrm{p}<0,05$. As análises foram realizadas com o programa estatístico SPSS, versão 16.0.

0 projeto de pesquisa foi submetido e aprovado pelo Comitê de Ética em Pesquisa do Hospital Universitário Lauro Wanderley, da Universidade Federal da Paraíba (Protocolo n. 04207). Após os esclarecimentos, os usuários assinaram o termo de consentimento livre e esclarecido, autorizando sua participação no estudo.

\section{Resultados}

A maioria da amostra (58,3\%) situava-se na faixa etária igual ou superior a 60 anos. Do total da amostra, 83,3\% eram do sexo feminino. Com relação ao estado civil, $66,6 \%$ dos usuários eram casados. Quanto ao tempo do diagnóstico do diabetes, $100 \%$ dos pesquisados apresentaram dois anos ou mais de diagnóstico. 0 tipo de diabetes mais recorrente foi a do tipo 2 (100\%). Com referência a presença de hipertensão arterial, 75,0\% da amostra apresentou associação com o diabetes. Em 37,5\% dos usuários, o edema estava presente nas extremidades inferiores.

Pelos dados do Quadro 1, a marcha com claudicação intermitente foi encontrada em 45,8\% dos usuários. Para $54,2 \%$ dos pacientes, a marcha foi realizada com a presença de dor. Em relação à distribuição do peso corporal, mais da metade dos pacientes $(58,3 \%)$ apresentaram alteração, sendo que em $33,3 \%$ a descarga foi mais acentuada para o lado direito. Quanto à base de apoio, 66,7\% não apresentavam alterações no apoio plantar.

De acordo com o Quadro 2 a sensibilidade dolorosa estava alterada em $8,4 \%$ dos pacientes. Em relação à sensibilidade térmica, apenas um paciente $(4,2 \%)$ não apresentou sensibilidade térmica dorsal, enquanto três $(12,5 \%)$ não apresentaram na região plantar.
Quadro 1 - Inspeção dinâmica da marcha dos usuários diabéticos

\begin{tabular}{lcc}
\hline Variáveis & $\begin{array}{c}\text { Total } \\
\mathbf{n}=\mathbf{2 4}\end{array}$ & $\%$ \\
\hline Marcha & 13 & 54,2 \\
Normal & 11 & 45,8 \\
Claudicante intermitente & & \\
Presença de dor na marcha & 13 & 54,2 \\
Sim & 11 & 45,8 \\
Não & & \\
Distribuição do peso corporal & 10 & 41,7 \\
Normal & 8 & 33,3 \\
Acentuado para o lado direito & 6 & 25,0 \\
Acentuado para o lado esquerdo & & \\
Base de apoio & & 33,3 \\
Normal & 8 & 37,5 \\
Supinação & 9 & 29,2 \\
Pronação & 7 & \\
\hline
\end{tabular}

Fonte: Dados da pesquisa.

Quadro 2 - Avaliação da sensibilidade e da resposta motora dos usuários diabéticos

(Continua)

\begin{tabular}{lrr}
\hline Variáveis & $\begin{array}{r}\text { Total } \\
\mathbf{n}=\mathbf{2 4}\end{array}$ & $\%$ \\
\hline Dolorosa & 1 & 4,2 \\
Algesia & 22 & 91,6 \\
Normal & 1 & 4,2 \\
Hiperestesia & & \\
Térmica dorsal & 23 & 95,8 \\
Sim & 1 & 4,2 \\
Não & & \\
Térmica plantar & 21 & 87,5 \\
Sim & 3 & 12,5 \\
Não & & \\
Tátil dorsal & 23 & 95,8 \\
Sim & 1 & 4,2 \\
Não & & \\
Tátil plantar & 22 & 91,6 \\
Sim & 2 & 8,4 \\
Não &
\end{tabular}


Quadro 2 - Avaliação da sensibilidade e da resposta motora dos usuários diabéticos

(Conclusão)

\begin{tabular}{lrr}
\hline Variáveis & $\begin{array}{c}\text { Total } \\
\mathbf{n}=\mathbf{2 4}\end{array}$ & $\%$ \\
\hline Protetora plantar & & \\
Positiva & 22 & 91,6 \\
Negativa & 2 & 8,4 \\
Vibratória & & \\
Presente & 22 & 91,6 \\
Ausente & 2 & 8,4 \\
\hline
\end{tabular}

Fonte: Dados da pesquisa.

Quanto à sensibilidade tátil, 4,2\% dos usuários não a possuíam na região dorsal e $8,4 \%$ na região plantar. Em 8,4\% dos usuários a sensibilidade protetora plantar não foi referida. A sensibilidade vibratória estava ausente em apenas $8,4 \%$ dos casos.

Os procedimentos adotados pelos usuários para o controle do diabetes tiveram um aumento estatisticamente significativo após a intervenção fisioterapêutica em relação à procura de profissionais adequados ( $p=0,004)$, a importância da mensuração frequente da glicose $(\mathrm{p}<0,001)$ e do controle alimentar $(\mathrm{p}=$ 0,017), conforme os dados do Quadro 3.

0 conhecimento da população estudada quanto à importância da adoção de hábitos saudáveis e de cuidados com os pés estão descritos no Quadro 4. Após a intervenção, ocorreram alterações estatisticamente significantes em relação ao hábito de andar sem calçado ( $p=0,003)$, a examinar os pés com frequência $(p=0,006)$, a não utilizar a prática do escalda-pé ( $p=0,013)$, a secar corretamente os pés $(p=0,016)$. Com relação ao uso do calçado adequado e do emprego da massagem terapêutica nos pés, da hidratação adequada e da detecção e solução de eventuais alterações nos pés, a intervenção teve um impacto relevante $(\mathrm{p}<0,001)$.

O Quadro 5 expressa o nível de conhecimento dos usuários quanto à importância da realização de exercícios para os pés. Após a intervenção, 100\% dos participantes consideraram benéficos os exercícios para os pés ( $\mathrm{p}<0,001)$, tornando-se um hábito frequente.

\section{Discussão}

0 diabetes mellitus é uma doença crônica que requer o monitoramento permanente da glicemia,

Quadro 3 - Procedimentos de controle do diabetes

(Continua)

\begin{tabular}{|c|c|c|c|c|c|}
\hline \multirow[b]{3}{*}{ Variáveis } & \multicolumn{5}{|c|}{ Intervenção } \\
\hline & \multicolumn{2}{|c|}{ Antes } & \multicolumn{2}{|c|}{ Depois } & \multirow[b]{2}{*}{$p$} \\
\hline & $\mathbf{n}$ & $\%$ & $\mathrm{n}$ & $\%$ & \\
\hline \multicolumn{6}{|l|}{ Procura do profissional } \\
\hline Sim & 17 & 70,8 & 24 & 100 & 0,004 \\
\hline Não & 1 & 4,2 & - & - & \\
\hline Às vezes & 3 & 25 & - & - & \\
\hline \multicolumn{6}{|l|}{ Ajuda da família } \\
\hline Sim & 11 & 45,8 & 11 & 45,8 & 1,00 \\
\hline Não & 2 & 8,4 & 2 & 8,4 & \\
\hline Às vezes & 11 & 45,8 & 11 & 45,8 & \\
\hline \multicolumn{6}{|l|}{ Medicamento } \\
\hline Sim & 10 & 41,7 & 15 & 62,5 & 0,15 \\
\hline Não & 2 & 8,3 & - & - & \\
\hline Às vezes & 12 & 50 & 9 & 37,5 & \\
\hline
\end{tabular}


Quadro 3 - Procedimentos de controle do diabetes

(Conclusão)

\begin{tabular}{|c|c|c|c|c|c|}
\hline \multirow[b]{3}{*}{ Variáveis } & \multicolumn{5}{|c|}{ Intervenção } \\
\hline & \multicolumn{2}{|c|}{ Antes } & \multicolumn{2}{|c|}{ Depois } & \multirow[b]{2}{*}{$p$} \\
\hline & $\mathrm{n}$ & $\%$ & $\mathrm{n}$ & $\%$ & \\
\hline \multicolumn{6}{|l|}{ Mensuração da glicose } \\
\hline Sim & 7 & 29,2 & 22 & 91,7 & $<0,001$ \\
\hline Não & 1 & 4,2 & - & - & \\
\hline Às vezes & 16 & 66,6 & 2 & 8,3 & \\
\hline \multicolumn{6}{|l|}{ Controle alimentar } \\
\hline Sim & 5 & 21,7 & 13 & 54,2 & 0,017 \\
\hline Não & 1 & 4,3 & - & - & \\
\hline Às vezes & 17 & 73,9 & 11 & 45,8 & \\
\hline
\end{tabular}

Fonte: Dados da pesquisa.

Quadro 4 - Hábitos e atitudes dos usuários diabéticos

(Continua)

\begin{tabular}{|c|c|c|c|c|c|}
\hline \multirow[b]{3}{*}{ Variáveis } & \multicolumn{5}{|c|}{ Intervenção } \\
\hline & \multicolumn{2}{|c|}{ Antes } & \multicolumn{2}{|c|}{ Depois } & \multirow[b]{2}{*}{$p$} \\
\hline & $\mathrm{n}$ & $\%$ & $\mathrm{n}$ & $\%$ & \\
\hline \multicolumn{6}{|l|}{ Andar sem calçados } \\
\hline Sim & 3 & 12,5 & - & - & 0,003 \\
\hline Não & 11 & 45,8 & 24 & 100 & \\
\hline Às vezes & 10 & 41,7 & - & - & \\
\hline \multicolumn{6}{|l|}{ Calçado adequado } \\
\hline Sim & 7 & 29,2 & 24 & 100 & $<0,001$ \\
\hline Não & 17 & 70,8 & - & - & \\
\hline Às vezes & - & - & - & - & \\
\hline \multicolumn{6}{|l|}{ Exame dos pés } \\
\hline Sim & 6 & 25,0 & 21 & 87,5 & 0,006 \\
\hline Não & 4 & 16,7 & - & - & \\
\hline Às vezes & 14 & 58,3 & 3 & 12,5 & \\
\hline \multicolumn{6}{|l|}{ Massagem terapêutica } \\
\hline Sim & 4 & 16,7 & 24 & 100 & \\
\hline Não & 20 & 83,3 & - & - & $<0,001$ \\
\hline Às vezes & - & - & - & - & \\
\hline \multicolumn{6}{|l|}{ Hidratação dos pés } \\
\hline Sim & 3 & 12,5 & 18 & 75,0 & \\
\hline Não & 2 & 8,3 & - & - & $<0,001$ \\
\hline Às vezes & 19 & 79,2 & 6 & 25,0 & \\
\hline
\end{tabular}


Quadro 4 - Hábitos e atitudes dos usuários diabéticos

(Conclusão)

\begin{tabular}{|c|c|c|c|c|c|}
\hline \multirow[b]{3}{*}{ Variáveis } & \multicolumn{5}{|c|}{ Intervenção } \\
\hline & \multicolumn{2}{|c|}{ Antes } & \multicolumn{2}{|c|}{ Depois } & \multirow[b]{2}{*}{$p$} \\
\hline & $n$ & $\%$ & $\mathrm{n}$ & $\%$ & \\
\hline \multicolumn{6}{|l|}{ Secagem dos pés } \\
\hline Sim & 9 & 37,5 & 19 & 79,2 & \\
\hline Não & 1 & 4,2 & - & - & $<0,001$ \\
\hline Às vezes & 14 & 58,3 & 5 & 20,8 & \\
\hline \multicolumn{6}{|l|}{ Prática do escalda-pé } \\
\hline Sim & 12 & 50,0 & . & - & \\
\hline Não & 2 & 8,3 & 22 & 91,7 & 0,016 \\
\hline Às vezes & 10 & 41,7 & 2 & 8,3 & \\
\hline \multicolumn{6}{|l|}{ Detecção de problemas } \\
\hline Sim & - & - & 24 & 100 & \\
\hline Não & 22 & 91,7 & - & - & $<0,001$ \\
\hline Às vezes & 2 & 8,3 & - & - & \\
\hline
\end{tabular}

Fonte: Dados da pesquisa.

Quadro 5 - Nível de conhecimento dos usuários sobre a prática de exercícios para os pés

\begin{tabular}{|c|c|c|c|c|c|}
\hline \multirow[b]{3}{*}{ Variáveis } & \multicolumn{5}{|c|}{ Intervenção } \\
\hline & \multicolumn{2}{|c|}{ Antes } & \multicolumn{2}{|c|}{ Depois } & \multirow[b]{2}{*}{$p$} \\
\hline & $n$ & $\%$ & $\mathrm{n}$ & $\%$ & \\
\hline \multicolumn{6}{|l|}{ Exercícios para os pés } \\
\hline Sim & 4 & 16,7 & 24 & 100 & \\
\hline Não & 16 & 66,6 & - & - & $<0,001$ \\
\hline Às vezes & 4 & 16,7 & - & - & \\
\hline \multicolumn{6}{|l|}{ Exercícios terapêuticos } \\
\hline Sim & - & - & 24 & 100 & \\
\hline Não & 24 & 100 & - & - & $<0,001$ \\
\hline Às vezes & - & - & - & - & \\
\hline \multicolumn{6}{|c|}{ Efeitos benéficos dos exercícios } \\
\hline \multicolumn{6}{|l|}{ Sim } \\
\hline Não & - & - & 24 & 100 & $<0,001$ \\
\hline Às vezes & 24 & 100 & - & - & \\
\hline
\end{tabular}

Fonte: Dados da pesquisa. 
mudanças no comportamento e a prática de hábitos saudáveis para seu controle. Desse modo, tornam-se indispensáveis ações e programas de intervenção para informar e conscientizar o diabético da importância do conhecimento sobre sua enfermidade, como parte integral do tratamento. Dessa forma, proporcionaria um melhor convívio com a doença, tornando-o protagonista de seu tratamento, reduzindo o ônus das condições crônicas e, assim, controlando a patologia e suas complicações, proporcionando-lhe uma melhor qualidade de vida $(11,12)$.

Pela análise das características sociodemográficas dos usuários, verificou-se um aumento da prevalência do diabetes mellitus à medida que a idade avançava. Mais de $2 / 3$ dos participantes (58,3\%) tinham mais de 60 anos. Resultados similares foram encontrados em estudos que obtiveram maior concentração de indivíduos diabéticos na faixa etária de 60 a 69 anos (13-15).

0 diabetes tipo 2 foi o mais recorrente (100\%). De acordo com a Sociedade Brasileira de Diabetes (2006) (6), o tipo detectado é o mais comumente encontrado, sendo diagnosticado em $90-95 \%$ dos casos. Provavelmente, a faixa etária dos participantes - a partir dos 40 anos - contribuiu para a obtenção do resultado. 0 DM tipo 2 é comumente diagnosticado em indivíduos com mais de 30 anos, aumentando sua prevalência com a idade, embora sua detecção possa ocorrer em qualquer faixa etária (16).

A hipertensão arterial sistêmica é uma comorbidade habitualmente encontrada em diabéticos, sendo mais frequente nesse grupo do que na população geral - afeta mais da metade desses indivíduos (17). Dentre os participantes do estudo, 75,0\% apresentaram hipertensão arterial, percentual superior ao encontrado em estudo sobre os fatores de risco para o DM tipo 2, no qual 66,6\% dos diabéticos eram também hipertensos (15). Cabe ressaltar que a hipertensão arterial associada ao DM multiplica o risco de morte cardíaca (16), e seu controle é capaz de reduzir de forma eficaz os óbitos relacionados ao diabetes, bem como as complicações microvasculares e a perda visual (14).

A doença vascular periférica (DVP) é bastante comum no diabético em decorrência do desenvolvimento da aterosclerose macrovascular. Em geral, os sintomas podem estar ausentes em repouso, entretanto, a claudicação intermitente poderá se manifestar quando a demanda pelo fluxo sanguíneo aumenta. Na fase mais avançada, os sintomas são dor em repouso, particularmente à noite, ulceração ou gangrena (18). No grupo estudado, $45,8 \%$ apresentaram marcha claudicante e $54,2 \%$ queixaram-se de dor durante a marcha (Quadro 1). Em estudos realizados, esses sintomas foram identificados em $10 \%$ e $15 \%$ dos indivíduos portadores de diabetes $(5,19)$.

Por meio da avaliação dinâmica da marcha dos usuários, foi possível mostrar que a prevalência de alterações na distribuição do peso corporal e da base de apoio, com consequente modificação do padrão da marcha, foram superiores a $58 \%$ e $67 \%$, respectivamente (Quadro 1). As alterações biomecânicas nas estruturas articulares, nos músculos e nos tendões do pé são frequentes em portadores de diabetes. Essas disfunções são decorrentes da neuropatia diabética (NPD) motora e da DVP, que, associadas, provocam hipotrofia muscular, acompanhada de fraqueza dos músculos estabilizadores das articulações metatarsofalangianas, provocando instabilidade e deformidades, além de alterar a biomecânica podal, resultando em pontos de pressão anormais e de atrito com o pé. Além de causar alterações na distribuição do peso corporal e da base de apoio, esses fatores estão associados ao aumento do risco de ulcerações e amputações (18-20).

Os participantes da intervenção não apresentaram alterações relevantes em relação à sensibilidade dolorosa, tátil, térmica, protetora e vibratória. As prevalências de deficits permaneceram abaixo dos 10\% (Quadro 2). Os resultados da intervenção estão em conformidade com um estudo que estimou o risco para problemas no pé diabético, por meio da avaliação da sensibilidade protetora plantar, em que $85 \%$ dos participantes apresentaram a sensibilidade preservada (21). É importante destacar que a avaliação da sensibilidade, a detecção precoce e o manejo da neuropatia diabética são importantes, visto que possibilita o tratamento específico nos casos sintomáticos, a orientação adequada e o acompanhamento das disfunções autonômicas relacionadas à maior morbimortalidade, além de proporcionar aos diabéticos o reconhecimento dos riscos de lesões nos membros inferiores $(5,18)$.

A mensuração regular da glicose caracteriza-se como um instrumento importante para a prevenção das complicações do diabetes. Inicialmente, menos de $30 \%$ dos usuários referiram realizar o monitoramento frequente da glicose. Após a intervenção fisioterapêutica, 91,7\% dos participantes passaram a realizar o controle glicêmico (Quadro 3). A importância da 
educação em saúde para o controle da glicemia foi mostrada por meio de um estudo em que a intervenção educativa reduziu os níveis de glicose dos pacientes (22). De acordo com o estudo, ficou consistentemente evidenciado que a melhora do controle glicêmico reduz o risco e a progressão das complicações microvasculares e neuropáticas, caracterizando-se como um recurso importante para a prevenção de úlceras e amputações (5).

Um dos pontos fundamentais no tratamento do diabetes é a educação alimentar, visto que não é possível um bom controle metabólico sem uma alimentação adequada. Além disso, o controle glicêmico pode reduzir a hemoglobina glicada entre $1-2 \%$ (23). No início do estudo, apenas cinco participantes $(21,7 \%)$ informaram que realizavam o controle alimentar. Depois da intervenção, dobrou o número de usuários que passaram a controlar a sua alimentação $(54,2 \%)$, contribuindo, assim, para a normalização da glicemia, a diminuição dos fatores de riscos cardiovasculares e a prevenção de complicações agudas e crônicas. Resultado semelhante a este estudo foi encontrado por Otero et al. (24), em pesquisa realizada para avaliar o conhecimento dos pacientes diabéticos acerca da doença, antes e depois da implementação de um programa de educação em diabetes, destacando-se, dentre outros, os cuidados com a alimentação (24).

A partir de 1975, a educação em diabetes vem sendo preconizada como indispensável no controle e no tratamento da doença. As práticas educativas em saúde, direcionadas a esse público e à sua família, têm como finalidade promover o conhecimento da patologia, o seu desenvolvimento, as comorbidades e o seu automanejo, como estratégia para prevenir as complicações e facilitar sua convivência com a doença (24). Embora sejam muitas as complicações sérias e dispendiosas que afetam os indivíduos com diabetes, as que afetam os pés representam das mais incapacitantes, advindas do mau controle da doença, representando cerca de 40 a $70 \%$ de todas as amputações das extremidades inferiores (18).

Foi marcante a mudança de hábitos e atitudes em relação ao autocuidado dos participantes do estudo, em decorrência da intervenção fisioterapêutica (Quadro 4). Todos os usuários (100\%) passaram a usar sapatos adequados e relataram haver deixado o hábito de andar sem eles, como também, uma parcela considerável de diabéticos passou a examinar, a hidratar, a secar e massagear os pés com frequência. Um estudo obteve resultados comparáveis aos deste, com o aumento do conhecimento dos diabéticos sobre a importância de cuidados e exame dos pés, após uma intervenção com ênfase na educação em saúde (24). 0 ato de massagear os pés promove uma vasodilatação, com consequente aumento da circulação local e sistêmica, além de reduzir o espasmo muscular e melhorar a capacidade de elasticidade do tecido conjuntivo. Todos esses efeitos têm influência benéfica sobre a neuropatia e a doença vascular do diabético (25). Cabe destacar que, por meio da intervenção fisioterapêutica, 91,7\% dos participantes passaram a não utilizar a prática do escalda-pé.

Com a intervenção fisioterapêutica (Quadro 5), foi possível verificar que todos os participantes (100\%) passaram a reconhecer a importância e os benefícios dos exercícios terapêuticos para a prevenção de úlceras nos pés, e todos os usuários (100\%) passaram a realizar esses exercícios ( $<<0,001)$. Embora sejam pouco frequentes os estudos destinados a avaliar o impacto da aplicação de exercícios para os pés em pacientes com comprometimento motor e sensorial, ocasionado pela neuropatia diabética, Goldsmith et al. (26) observaram uma redução da pressão plantar e, consequentemente, redução da formação de úlceras, após um mês de realização de exercícios. Gomes et al. (27) atenuaram os efeitos da sintomatologia da neuropatia periférica em diabéticos, durante o período de intervenção, com exercícios terapêuticos. De acordo com os autores, os exercícios promoveram um melhor aporte sanguíneo para os membros inferiores e, dessa forma, podem ter contribuído para a cicatrização dos ferimentos, a redução da hipóxia endoneural e melhoria da condução nervosa.

Com base no exposto, pode-se afirmar que o investimento em ações de educação em saúde torna-se cada vez mais necessário, a fim de que os portadores de diabetes possam ter um maior domínio e compreensão da importância do autocuidado, com ênfase nos cuidados básicos com os pés.

Neste estudo, evidenciou-se que a intervenção fisioterapêutica, com as ações em educação em saúde, foi um instrumento importante para conscientizar os participantes e modificar hábitos e atitudes que colocavam em risco o pé dos usuários.

Portanto, é de fundamental importância que as políticas públicas de saúde, particularmente na atenção básica de saúde, priorizem ações multidisciplinares, de modo que o cuidado com os pés dos diabéticos seja contemplado, aliado a um bom controle glicêmico e ao estímulo à prática de exercícios físicos. 


\section{Agradecimentos}

0 estudo recebeu financiamento do Conselho Nacional de Desenvolvimento Científico e Tecnológico (CNPq), Edital Universal MCT/CNPq n. 015/2007, processo n. 474301/2007-9.

\section{Referências}

1. Sociedade Brasileira de Diabetes. Consenso brasileiro sobre diabetes 2002: diagnóstico e classificação do diabetes melito e tratamento do diabetes melito do tipo 2. Rio de Janeiro: Diagraphic; 2003.

2. World Diabetes Foundation (WDF). A supplement to practical diabetes international. A Report from the diabetes summit for Latin America, 2010. Salvador: WDF; 2010.

3. World Health Organization (WHO). 2008-2013 Action plan for the global strategy for the prevention and control of noncommunicable diseases: prevent and control cardiovascular diseases, cancers, chronic respiratory diseases and diabetes. Geneva; 2009.

4. Barceló AC, Rajpathak S, Robles S. 0 custo do diabetes na América Latina e no Caribe. Bull WHO. 2003;81:1927. PMid:12640472.

5. Ochoa-Vigo K, Torquato MT, Silvério IA, Queiroz FA, de-la-Torre-Ugarte-Guanilo MC, Pace AE. Caracterização de pessoas com diabetes em unidades de atenção primária e secundária em relação a fatores desencadeantes do pé diabético. Acta Paul Enferm. 2006;19(3):29603. doi:10.1590/S0103-21002006000300007.

6. Sociedade Brasileira de Diabetes. Diretrizes da sociedade brasileira de diabetes: tratamento e acompanhamento do diabetes mellitus; 2006.

7. Gamba MA, Gotlieb S, Bergamaschi DP, Vianna LA. Amputações de extremidades inferiores por diabetes mellitus: estudo caso-controle. Rev Saúde Pública. 2004;38(3):399-4. doi:10.1590/ S0034-89102004000300010.

8. Mendonça SS, Morais JS, Moura MCGG. Proposta de um protocolo de avaliação fisioterapêutica para os pés de diabéticos. Fisioter Mov. 2011;24(2):285-98.

9. Barbui EC, Cocco MI. Conhecimento do cliente diabético em relação aos cuidados com os pés. Rev Esc Enferm USP. 2002;36(1):97-103. doi:10.1590/ S0080-62342002000100014.
10. Nascimento LMO, Damasceno MMC, Marques RLL, Silva LF, Montenegro RM, Almeida PC. Avaliação dos pés de diabéticos: estudo com pacientes de um hospital universitário. Texto Contexto Enferm. 2004;13(1):63-73.

11. Organização Mundial da Saúde (OMS). Cuidados inovadores para condições crônicas: componentes estruturais de ação. Relatório Mundial; 2003.

12. Biral AM, Cardoso PM, Grunspan S. A importância do educador em diabetes mellitus. Diabetes Clinic. 2005;9(3):193-9.

13. Souza LJ, Chalita FEB, Reis AFF, Teixeira CL, Gicovate Neto C, Bastos DA, et al. Prevalência de diabetes mellitus e fatores de risco em Campos dos Goytacazes, RJ. Arq Bras Endocrinol Metab. 2003;47(1)69-4. doi:10.1590/S0004-27302003000100011.

14. Grillo MF, Gorini MI. Caracterização de pessoas com diabetes mellitus tipo 2. Rev Bras Enferm. 2007;60(1):4954. doi:10.1590/S0034-71672007000100009.

15. Carolino ID, Molena-Fernandes CA, Tasca RS, Marcon SS, Cuman RK. Fatores de risco em pacientes com diabetes mellitus tipo 2. Rev Latino-Am Enfermagem. 2008;16(2):238-44. doi:10.1590/ S0104-11692008000200011.

16. Brasil. Ministério da Saúde. Departamento de Atenção Básica. Área técnica de diabetes e hipertensão arterial. Hipertensão arterial sistêmica e diabetes mellitus - protocolo. Cad Atenção Básica. 2001;96(7):1-33.

17. Gus I, Fischmann A, Medina C. Prevalence of risk factors for coronary artery disease in the Brazilian State of Rio Grande do Sul. Arq Bras Cardiol. 2002;78(5):48490. doi:10.1590/S0066-782X2002000500005.

18. Brasil. Ministério da Saúde. Secretaria de Estado de Saúde do Distrito Federal. Grupo de trabalho internacional sobre pé diabético. Consenso internacional sobre pé diabético. Brasília: Ministério da Saúde; 2001.

19. Rocha RM, Zanetti ML, Santos MA. Comportamento e conhecimento: fundamentos para prevenção do pé diabético. Acta Paul Enferm. 2009;22(1):17-23. doi:10.1590/S0103-21002009000100003.

20. Sumpio BE. Foot ulcers. New Engl J Med. 2006;343(11): 787-93. doi:10.1056/NEJM200009143431107. 
21. Sims Jr DS, Cavanagh PR, Ulbrecht JS. Risk factors in the diabetic foot. Recognition and management. Phys Ther. 1988;68(12):1887-902. PMid:3057524.

22. Silva TR, Feldmam C, Lima MHA, Nobre MRC, Domingues RZL. Controle de diabetes mellitus e hipertensão arterial com grupos de intervenção educacional e terapêutica em seguimento ambulatorial de uma unidade básica de saúde. Saúde e Sociedade. 2006;15(3)180-89. doi:10.1590/ S0104-12902006000300015.

23. Brasil. Ministério da Saúde. Secretaria de Atenção à Saúde. Departamento de Atenção Básica. Cadernos de atenção básica. Diabetes mellitus. Brasília: Ministério da Saúde; 2006.

24. Otero LM, Zanetti ML, Ogrizio MD. Conhecimento do paciente diabético a cerca de sua doença, antes e depois da implementação de um programa de educação em diabetes. Rev Latino-am Enfermagem. 2008;16(2):1-7.
25. Simons JG, Travell LS. Dor e disfunção miofascial: manual dos pontos-gatilho. 2. ed. Porto Alegre: Artmed; 2005.

26. Goldsmith JR, Lidtke RH, Shott S. The effects of range-of-motion therapy on the plantar pressures of patients with diabetes mellitus. J Am Podiatr Med Assoc. 2002;92(9):483-90. PMid:12381797.

27. Gomes AA, Sartor CD, João SMA, Sacco ICN, Bernik MMS. Efeitos da intervenção fisioterapêutica nas respostas sensoriais e funcionais de diabéticos neuropatas. Fisioter Pesq. 2007;14(1):14-21.

Recebido: 28/06/2011

Received: 06/28/2011

Aprovado: 16/01/2012

Approved: 01/16/2012 